\title{
Impacts of geological heterogeneity and artificial decolmation on hydrogeochemistry during bank filtration
}

\author{
WEISHI WANG ${ }^{1, *}$, BIN HU ${ }^{2}$, HERMANN-JOSEF \\ LENSING $^{3}$, DANIEL STRASSER ${ }^{3}$, RUIHUI CHEN $^{4}$ \\ AND MATTHIAS MUNZ ${ }^{1}$ \\ ${ }^{1}$ University of Potsdam, Karl-Liebknecht-St. 24-25, 14476, \\ Potsdam, Germany \\ (*Correspondences: wangwawu@gmail.com) \\ ${ }^{2}$ Research Center for Eco-Environmental Science Chinese \\ Academy of Sciences, Beijing 100085, China \\ ${ }^{3}$ Department of Geotechnical Engineering, BAW, \\ Kussmaulstraße 17, 76187, Karlsruhe, Germany \\ ${ }^{4}$ Beijing Normal Univ, Beijing 100875, China
}

Bank filtration (BF) is a technology for drinking water withdrawal extensively applied all over the world ${ }^{[1]}$. There is a debate of the potential effects of declogging on waterworks treatment efficiency in the BF sites, especially for geological heterogeneity sites ${ }^{[2]}$. Moreover, the temporal and spatial variations in the redox environment after artificial decolmation at $\mathrm{BF}$ sites with substantial geological heterogeneity has not been systemically studied. Thus, the objective of this study is to explore effects of artificial decolmation on hydrogeochemical characteristics and water quality at a BF site characterized by geological heterogeneity.

The study area locates at a BF waterwork in the south of a human-made canal in Potsdam, Germany. It was built on a highly heterogeneous distribution of glacial tills deposits ${ }^{[3]}$. According to the sampling analysis results, the geological heterogeneity has significant impacts on groundwater flow patterns, which further induces discrepancy of redox zonation in the near-bank wells after decolmation. Overall, after reconstruction, the redox condition has been changed locally in the nearbank wells (about 13.2 16.7 $\mathrm{m}$ to the cannal), but buffered by the long travel time, remained constantin the distant wells. The infiltration pathway is more than $126 \mathrm{~m}$, which is long enough to act as an efficient buffer for the drastic change caused by the reconstruction, leading to no decline in the water quality indices (DOC, SUVA, redox zonation) of the $\mathrm{BF}$ site after the decolmation works.

[1] Hu et al., $2016 \mathrm{JH}, 895-899$

[2] Ascott et al., 2016 STE, 554, 89-101

[3] Wang et al., 2019 HJ, 1-21 